\title{
AN ENTIRE FUNCTION WITH NO FIXED POINTS AND NO INVARIANT BAKER DOMAINS
}

\author{
WALTER BERGWEILER
}

\begin{abstract}
We show that there exists an entire function which has neither fixed points nor invariant Baker domains. The question whether such a function exists was raised by Buff.
\end{abstract}

\section{INTRODUCTION AND RESULT}

Let $f$ be a meromorphic function and denote by $f^{n}$ the $n$-th iterate of $f$. An invariant component $U$ of the Fatou set of $f$ such that $\left.f^{n}\right|_{U} \rightarrow \infty$ as $n \rightarrow \infty$ is called an invariant Baker domain; cf. [3, §4.7] or [11]. It was suggested by Douady that invariant Baker domains of the Newton function $f(z)=z-g(z) / g^{\prime}(z)$ of an entire function $g$ are related to paths where $g$ tends to the asymptotic value 0 . In response to Douady's question it was shown in [8] that under mild additional hypotheses the existence of an invariant Baker domain does indeed imply that 0 is an asymptotic value of $g$. However, this is not always the case [5].

If $g$ has no zeros at all, then the Newton function $f$ has no fixed points. Moreover, 0 is an asymptotic value of $g$ by Iversen's theorem [10, p. 289]. This led Buff to ask whether there exists an entire function having no fixed points and no invariant Baker domains. We show that such a function exists.

Theorem. There exists an entire function with no fixed points and no invariant Baker domains.

A meromorphic function with this property was constructed in [4]. The present construction is based on similar ideas. As in [4], a function $f$ satisfying the conclusion of the theorem can be given explicitly.

Let $\left(r_{k}\right)$ be a sequence of real numbers tending to $\infty$ and let $\left(n_{k}\right)$ be a sequence of positive integers satisfying $n_{k} \geq k$ for all $k \in \mathbb{N}$. Then

$$
h(z)=\prod_{k=1}^{\infty}\left(1+\left(\frac{z}{r_{k}}\right)^{n_{k}}\right)
$$

defines an entire function $h$. Indeed, if $|z| \leq R$ and $k$ is so large that $r_{k} \geq 2 R$, then $\left|z / r_{k}\right|^{n_{k}} \leq 2^{-k}$, implying that the infinite product converges locally uniformly.

For $k \geq 2$ we put $m_{k}=\sum_{j=1}^{k-1} n_{j}$. We shall show that if

$$
r_{k} \geq 2 r_{k-1} \geq 4 \quad \text { and } \quad n_{k} \geq 20 r_{k}^{2} \exp \left(4 r_{k}^{m_{k}}\right)
$$

for $k \geq 2$, then $f(z)=z+e^{h(z)}$ has the required property.

Acknowledgment. I thank Alexandre Eremenko for useful comments.

2010 Mathematics Subject Classification. Primary 37F10; Secondary 30D05, 49M15, 65H05.

Supported by the ESF Research Networking Programme HCAA. 


\section{Preliminaries}

2.1. The hyberbolic metric. We need some standard results about the hyperbolic metric which can be found in, e.g., [9, Section I.4].

We denote the open disk of radius $r$ around a point $c \in \mathbb{C}$ by $D(c, r)$ and put $\mathbb{D}=D(0,1)$. The density of the hyperbolic metric in a hyperbolic domain $U$ is denoted by $\lambda_{U}$, normalized such that $\lambda_{\mathbb{D}}(z)=2 /\left(1-|z|^{2}\right)$. The hyperbolic metric is denoted by $\rho_{U}$. For $a, b \in U$ we thus have

$$
\rho_{U}(a, b)=\inf _{\gamma} \int_{\gamma} \lambda_{U}(z)|d z|
$$

where the infimum is taken over all curves $\gamma$ that connect $a$ and $b$. Then $[9$, p. 11]

$$
\rho_{\mathbb{D}}(0, z)=\log \frac{1+|z|}{1-|z|} \quad \text { for } z \in \mathbb{D}
$$

It follows from Schwarz's lemma and the Koebe one quarter theorem that if $U$ is simply connected, then [9, Theorem I.4.3]

$$
\frac{1}{2 \operatorname{dist}(z, \partial U)} \leq \lambda_{U}(z) \leq \frac{2}{\operatorname{dist}(z, \partial U)}
$$

for all $z \in U$. Here $\operatorname{dist}(z, \partial U)=\inf _{\zeta \in \partial U}|\zeta-z|$.

The following lemma is a simple consequence of (2.2).

Lemma 1. Let $U$ be a simply connected hyperbolic domain, $a, b \in U$ and $c \in \mathbb{C} \backslash U$. Then

$$
\rho_{U}(a, b) \geq \frac{1}{2}|\log | \frac{b-c}{a-c}|| .
$$

Proof. Without loss of generality we may assume that $c=0$. Let $\gamma$ be a curve from $a$ to $b$ and let $L$ be a branch of the logarithm defined in $U$. Then (2.2) yields

$$
\begin{aligned}
\int_{\gamma} \lambda_{U}(z)|d z| & \geq \frac{1}{2} \int_{\gamma} \frac{|d z|}{\operatorname{dist}(z, \partial U)} \geq \frac{1}{2} \int_{\gamma} \frac{|d z|}{|z|} \geq \frac{1}{2}\left|\int_{\gamma} \frac{d z}{z}\right| \\
& =\frac{1}{2}|L(b)-L(a)| \geq \frac{1}{2}|\operatorname{Re}(L(b)-L(a))|=\frac{1}{2}|\log | \frac{b}{a}||,
\end{aligned}
$$

from which the conclusion follows.

The next lemma follows easily from (2.1) and the triangle inequality.

Lemma 2. If $a, b \in D(c, r / 2)$, then $\rho_{D(c, r)}(a, b) \leq 2 \log 3$.

Finally we have the following form of Schwarz's lemma [9, Theorem I.4.3].

Lemma 3. Let $U, V$ be hyperbolic domains, $f: U \rightarrow V$ holomorphic and $a, b \in U$. Then $\rho_{V}(f(a), f(b)) \leq \rho_{U}(a, b)$.

Applying this lemma to $f(z)=z$ yields

$$
\rho_{V}(a, b) \leq \rho_{U}(a, b) \quad \text { if } U \subset V .
$$


2.2. Some growth estimates. We have to estimate the growth of $h$ on certain circles from above and below. For $k \geq 2$ and $|z|=r_{k}$ we have

$$
\begin{aligned}
\log |h(z)| & \leq \sum_{j=1}^{k-1} \log \left(1+\left(\frac{r_{k}}{r_{j}}\right)^{n_{j}}\right)+\log 2+\sum_{j=k+1}^{\infty} \log \left(1+\left(\frac{r_{k}}{r_{j}}\right)^{n_{j}}\right) \\
& \leq \sum_{j=1}^{k-1} \log \left(1+\frac{1}{2} r_{k}{ }^{n_{j}}\right)+\log 2+\sum_{j=k+1}^{\infty}\left(\frac{r_{k}}{r_{j}}\right)^{n_{j}} \\
& \leq \sum_{j=1}^{k-1} \log \left(r_{k}{ }^{n_{j}}\right)+\log 2+\sum_{j=k+1}^{\infty} 2^{-n_{j}} \leq m_{k} \log r_{k}+2 \log 2 .
\end{aligned}
$$

Hence

$$
|h(z)| \leq 4 r_{k}^{m_{k}} \quad \text { for }|z|=r_{k}
$$

and $k \geq 2$.

We put $s_{k}=\left(1+1 / n_{k}\right) r_{k}$. For $t \in[0,2 \pi]$ and $z=s_{k} e^{i t}$ we have

$$
\begin{aligned}
h(z) & =\prod_{j=1}^{k-1}\left(1+\left(\frac{z}{r_{j}}\right)^{n_{j}}\right) \cdot\left(1+\left(1+\frac{1}{n_{k}}\right)^{n_{k}} e^{i n_{k} t}\right) \cdot \prod_{j=k+1}^{\infty}\left(1+\left(\frac{z}{r_{j}}\right)^{n_{j}}\right) \\
& \sim \prod_{j=1}^{k-1}\left(\frac{z}{r_{j}}\right)^{n_{j}}\left(1+e \cdot e^{i n_{k} t}\right)=\prod_{j=1}^{k-1}\left(\frac{s_{k}}{r_{j}}\right)^{n_{j}} e^{i m_{k} t}\left(1+e \cdot e^{i n_{k} t}\right)
\end{aligned}
$$

as $k \rightarrow \infty$. Putting

$$
T_{k}=\prod_{j=1}^{k-1}\left(\frac{s_{k}}{r_{j}}\right)^{n_{j}}
$$

we thus have

$$
h\left(s_{k} e^{i t}\right) \sim T_{k} e^{i m_{k} t}\left(1+e \cdot e^{i n_{k} t}\right)
$$

as $k \rightarrow \infty$.

It is not difficult to see that for each $\varphi \in \mathbb{R}$ there exists $\theta=\theta(\varphi) \in[0,1]$ such that $e^{2 \pi i \varphi}\left(1+e \cdot e^{2 \pi i \theta}\right)$ is positive. For $\nu \in\left\{0,1, \ldots, n_{k}-1\right\}$ we put

$$
\theta_{\nu}=\theta\left(\nu m_{k} / n_{k}\right) \quad \text { and } \quad p_{\nu}=e^{2 \pi i \nu m_{k} / n_{k}}\left(1+e \cdot e^{2 \pi i \theta_{\nu}}\right) \text {. }
$$

Then $p_{\nu}$ is positive and thus $p_{\nu}=\left|p_{\nu}\right| \geq e-1$. Since $m_{k} / n_{k} \rightarrow 0$ by (1.1), we deduce from (2.6) that

$$
h\left(s_{k} e^{2 \pi i\left(\nu+\theta_{\nu}\right) / n_{k}}\right) \sim T_{k} e^{2 \pi i\left(\nu+\theta_{\nu}\right) m_{k} / n_{k}}\left(1+e \cdot e^{2 \pi i\left(\nu+\theta_{\nu}\right)}\right)=p_{\nu} T_{k} e^{2 \pi i \theta_{\nu} m_{k} / n_{k}} \sim p_{\nu} T_{k}
$$

Thus

$$
\operatorname{Re} h\left(s_{k} e^{2 \pi i\left(\nu+\theta_{\nu}\right) / n_{k}}\right) \geq T_{k}
$$

for large $k$ and all $\nu \in\left\{0,1, \ldots, n_{k}-1\right\}$. 


\section{Proof of the theOREM}

Let $f$ be as defined in the introduction and suppose that $f$ has an invariant Baker domain $U$. By a result of Baker [1], $U$ is simply connected. Take $z_{0} \in U$, connect $z_{0}$ and $f\left(z_{0}\right)$ by a curve $\gamma_{0}$ in $U$ and put $\gamma=\bigcup_{j=0}^{\infty} f^{j}\left(\gamma_{0}\right)$. Then $\gamma$ is a curve in $U$ connecting $z_{0}$ to $\infty$. As $\gamma_{0}$ is compact, there exists $K>0$ such that $\rho(f(z), z) \leq K$ for all $z \in \gamma_{0}$. Since every $z \in \gamma$ has the form $z=f^{j}(\zeta)$ for some $\zeta \in \gamma_{0}$ and some $j \geq 0$, Lemma 3 yields

$$
\rho(f(z), z) \leq K \quad \text { for } z \in \gamma .
$$

For large $k$ the curve $\gamma$ intersects the circle $\left\{z:|z|=r_{k}\right\}$. Let $z_{k}$ be a point of intersection.

We shall show first that if $k$ is large enough, then the disk $D\left(z_{k}, 20 r_{k} / n_{k}\right)$ is not contained in $U$; that is,

$$
D\left(z_{k}, 20 r_{k} / n_{k}\right) \cap \partial U \neq \emptyset .
$$

In order to do so we assume that $D\left(z_{k}, 20 r_{k} / n_{k}\right) \subset U$. We write $z_{k}=r_{k} e^{2 \pi i t_{k}}$ with $t_{k} \in[0,1)$ and put $\nu=\left[n_{k} t_{k}\right]$, where $[x]$ denotes the largest integer not greater than $x$. Thus $n_{k} t_{k}=\nu+\delta$ where $\nu \in\left\{0,1, \ldots, n_{k}-1\right\}$ and $\delta \in[0,1)$. Let

$$
a_{k}=r_{k} e^{(2 \nu+1) \pi i / n_{k}} \quad \text { and } \quad b_{k}=s_{k} e^{2 \pi i\left(\nu+\theta_{\nu}\right) / n_{k}} .
$$

Then

$$
\left|a_{k}-z_{k}\right|=r_{k}\left|e^{(2 \nu+1) \pi i / n_{k}-2 \pi i t_{k}}-1\right|=r_{k}\left|e^{(1-2 \delta) \pi i / n_{k}}-1\right| \sim \frac{|1-2 \delta| \pi r_{k}}{n_{k}}
$$

and

$$
\begin{aligned}
\left|b_{k}-z_{k}\right| & \leq\left|b_{k}-r_{k} e^{2 \pi i\left(\nu+\theta_{\nu}\right) / n_{k}}\right|+\left|r_{k} e^{2 \pi i\left(\nu+\theta_{\nu}\right) / n_{k}}-z_{k}\right| \\
& =s_{k}-r_{k}+r_{k}\left|e^{2 \pi i\left(\nu+\theta_{\nu}\right) / n_{k}-2 \pi i t_{k}}-1\right| \\
& =\frac{r_{k}}{n_{k}}+r_{k}\left|e^{2 \pi i\left(\theta_{\nu}-\delta\right) / n_{k}}-1\right| \\
& \sim \frac{\left(1+2 \pi\left|\theta_{\nu}-\delta\right|\right) r_{k}}{n_{k}}
\end{aligned}
$$

which implies that

$$
a_{k} \in D\left(z_{k}, 10 r_{k} / n_{k}\right) \quad \text { and } \quad b_{k} \in D\left(z_{k}, 10 r_{k} / n_{k}\right)
$$

for large $k$. Lemma 2 and (2.3) now yield

$$
\rho_{U}\left(a_{k}, b_{k}\right) \leq \rho_{D\left(z_{k}, 20 r_{k} / n_{k}\right)}\left(a_{k}, b_{k}\right) \leq 2 \log 3 .
$$

Since $h\left(a_{k}\right)=0$ by the definition of $h$ and $\operatorname{Re} h\left(b_{k}\right) \geq T_{k} \geq s_{k} / r_{1}$ by (2.5) and (2.7), we have

$$
\left|f\left(a_{k}\right)\right|=\left|a_{k}+1\right| \leq r_{k}+1 \quad \text { and } \quad\left|f\left(b_{k}\right)\right| \geq e^{s_{k} / r_{1}}-s_{k} \geq s_{k}^{2} \geq r_{k}^{2}
$$

for large $k$. Fix a point $c \in \partial U$. Lemma 1 and (3.4) imply that

$$
\rho_{U}\left(f\left(a_{k}\right), f\left(b_{k}\right)\right) \geq \frac{1}{2} \log \left|\frac{f\left(b_{k}\right)-c}{f\left(a_{k}\right)-c}\right| \geq \frac{1}{2} \log \frac{r_{k}^{2}-|c|}{r_{k}+1+|c|}
$$

for large $k$. Now a contradiction is obtained from Lemma 3 , (3.3) and (3.5), provided $k$ is sufficiently large. This contradiction shows that (3.2) holds for large $k$. 
Thus, for large $k$, there exists $c_{k} \in D\left(z_{k}, 20 r_{k} / n_{k}\right) \cap \partial U$. Lemma 1 now yields

$$
\rho_{U}\left(f\left(z_{k}\right), z_{k}\right) \geq \frac{1}{2} \log \left|\frac{f\left(z_{k}\right)-c_{k}}{z_{k}-c_{k}}\right|=\frac{1}{2} \log \left|\frac{e^{h\left(z_{k}\right)}}{z_{k}-c_{k}}+1\right| .
$$

Since

$$
\left|\frac{e^{h\left(z_{k}\right)}}{z_{k}-c_{k}}\right| \geq \frac{e^{-\left|h\left(z_{k}\right)\right|}}{\left|z_{k}-c_{k}\right|} \geq \frac{n_{k} \exp \left(-4 r_{k}^{m_{k}}\right)}{20 r_{k}} \geq r_{k}
$$

for $k \geq 2$ by (1.1) and (2.4), we obtain

$$
\rho_{U}\left(f\left(z_{k}\right), z_{k}\right) \geq \frac{1}{2} \log \left(r_{k}-1\right)
$$

for large $k$, contradicting (3.1).

Remark 1. Buff and Rückert [8] considered virtual immediate basins instead of invariant Baker domains. However, for functions for which all Baker domains are simply connected the two concepts coincide; cf. the discussion in [4, p. 431] or [8, p. 4]. By the result of Baker [1] already quoted, this holds in particular for entire functions. By a recent result of Barański, Fagella, Jarque and Karpińska [2], it also holds for Newton maps of entire functions.

Remark 2. The function $f$ constructed in the proof is the Newton function for

$$
g(z)=\exp \left(-\int_{0}^{z} e^{-h(t)} d t\right) .
$$

Since $g$ has no zeros, $g$ has a direct singularity over 0; see [10, p. 289] for this result, as well as [6, 12, for the terminology used here and below. As $f$ has no invariant Baker domains, $g$ has no logarithmic singularity over 0 by one of the results obtained by Buff and Rückert in the paper already mentioned in the introduction [8, Theorem 4.1]. Thus $g$ has a direct non-logarithmic singularity over 0 . This implies [7, Theorem 5] that $g$ has uncountably many direct non-logarithmic singularities over 0. As $g$ has no critical points, a result of Sixsmith [12, Theorem 1.2] yields that every neighborhood of any of these singularities contains a neighborhood of an indirect or logarithmic singularity of $g$ whose projection is different from 0 . Overall we see that the set of singularities of the inverse of $g$ has a quite complicated structure.

\section{REFERENCES}

[1] I. N. Baker, The domains of normality of an entire function. Ann. Acad. Sci. Fenn. Ser. A I Math. 1 (1975), 277-283.

[2] Krzysztof Barański, Núria Fagella, Xavier Jarque and Bogusława Karpińska, On the connectivity of the Julia sets of meromorphic functions. Preprint, arXiv: 1206.6667.

[3] Walter Bergweiler, Iteration of meromorphic functions. Bull. Amer. Math. Soc. (N. S.) 29 (1993), 151-188.

[4] Walter Bergweiler, Newton's method and Baker domains. J. Difference Equ. Appl. 16 (2010), 427-432.

[5] Walter Bergweiler, David Drasin and James K. Langley, Baker domains for Newton's method. Ann. Inst. Fourier 57 (2007), 803-814.

[6] Walter Bergweiler and Alexandre Eremenko, On the singularities of the inverse to a meromorphic function of finite order. Rev. Mat. Iberoamericana 11 (1995), 355-373. 
[7] Walter Bergweiler and Alexandre Eremenko, Direct singularities and completely invariant domains of entire functions. Illinois J. Math. 52 (2008), 243-259.

[8] Xavier Buff and Johannes Rückert, Virtual immediate basins of Newton maps and asymptotic values. Int. Math. Res. Not. 2006, 65498, 1-18.

[9] Lennart Carleson and Theodore W. Gamelin, Complex Dynamics. Universitext: Tracts in Mathematics. Springer-Verlag, New York, 1993.

[10] Rolf Nevanlinna, Eindeutige analytische Funktionen. Springer-Verlag, Berlin, Göttingen, Heidelberg, 1953.

[11] P. J. Rippon, Baker domains. In "Transcendental Dynamics and Complex Analysis". London Math. Soc. Lect. Note Ser. 348. Edited by P. J. Rippon and G. M. Stallard, Cambridge University Press, Cambridge, 2008, 371-395.

[12] D. J. Sixsmith, A new characterisation of the Eremenko-Lyubich class. Preprint, arXiv: 1205.1976.

Mathematisches Seminar, Christian-Albrechts-Universität ZU Kiel, LudewigMeyn-Str. 4, D-24098 Kiel, Germany

E-mail address: bergweiler@math.uni-kiel.de 